Abstract E-131 Table 2 Baseline characteristics, timings and outcomes in patients undergoing Mechanical Thrombectomy (MT) for large vessel occlusion.

\begin{tabular}{lll}
\hline & $\begin{array}{l}\text { Natives } \\
(\mathrm{N}=7)\end{array}$ & $\begin{array}{l}\text { Comparison Group } \\
(\mathrm{N}=28)\end{array}$ \\
\hline Clinical baseline & 13 & 15.5 \\
- Median NIHSS & 15 & 15 \\
- Median GCS & 0 & 0 \\
- Median Pre-stroke mRS & $4(57 \%)$ & $18(62 \%)$ \\
- Treatment with t-PA (\%) & & \\
Timing & 170 & 220 \\
- Median LKW - UNM arrival (min) & 88 & 49 \\
- Median UNM arrival - Groin puncture (min) & 296 & 263 \\
- Median LKW - Groin puncture (min) & 348 & 346 \\
- Median LKW- Reperfusion time (min) & & \\
Outcomes & 1 & 2 \\
- Median mRS at 6 months & 2 & 9 \\
- Median NIHSS at discharge & $7(100 \%)$ & $23(82 \%)$ \\
- Successful TICl score 2b or 3 (\%) & $1(14 \%)$ & $5(18 \%)$ \\
- Hemorrhagic transformation (\%) & $1(14 \%)$ & $1(4 \%)$ \\
- Mortality (\%) & & \\
\hline
\end{tabular}

Conclusion This is the first ever descriptive study evaluating outcomes in NA patients undergoing MT. Prevalence of vascular risk factors in NA was noticeably higher compared to the comparison group. Our retrospective study showed that Native American patients similar functional and neurological outcomes for thrombectomy.

Disclosures A. Ikram: None. S. Suriya: None. M. Farooqui: None. O. Owens: None. A. Alvarado Arias: None. D. Sorte: None. A. Carlson: None. S. Ortega-Guiterrez: None. M. Torbey: None. A. Zafar: None.

\section{E-132 USING CUTTING-EDGE TECHNOLOGY TO REINFORCE BEST PRACTICE STROKE CARE}

S Lang ${ }^{*}$. Corazon, Inc., Pittsburgh, PA

\subsection{6/neurintsurg-2019-SNIS.207}

Introduction/Purpose There continues to be a lack of standard data management tools for neurovascular service lines and stroke programs. Many hospitals use 'home-grown' spreadsheets or upload to various registries which are designed more toward research initiatives than daily operations.

Installation of a dedicated Neurovascular Information System (NVIS) will result in improved efficiency, quality, and financial performance in operating a stroke program and/or neurovascular service line.

Materials and methods A small rural hospital in Pennsylvania installed a NVIS for daily use within its Primary Stroke Center. The NVIS was designed to support data entry and capture clinical data for process improvement, care standardization, and support stoke program growth. A commitment to move from retrospective data collection to near real-time data collection and analysis capitalized on the ability to adjust care for the next patient. The data from the NVIS was used to amplify opportunities for quality improvement and identify flaws in policies/procedures, care standards, and other program components. An effort to reinforce best practice standards was initiated based on findings from the data.

Results The stroke care team used the data from the NVIS to identify and quickly and efficiently fix issues real-time versus addressing any problems months later after waiting for formal data registry reports. The following results were realized: an increased swallow screen compliance, increased use of the NIH assessment by ED physicians and front-line staff, an increase in t-PA utilization rate, an increase in the number of patients receiving t-PA within 60 minutes of arrival, an increase in the number of discharges to home, and an increase in the number of patients arriving by ambulance. Additionally, the chart abstraction times were decreased.

Conclusion Utilization of a dedicated NVIS reversed the narrative related to stroke program management and allowed program leaders to use this clinical infrastructure and accurate data to drive practice improvement and assist on-site staff to more effectively manage the care continuum from pre-admission through post-discharge.

Disclosures S. Lang: None.

\section{E-133 ADHERENCE TO ENDOVASCULAR TREATMENT GUIDELINES IN ACUTE ISCHEMIC STROKE: INSIGHTS FROM AN INTERNATIONAL MULTIDISCIPLINARY SURVEY}

${ }^{1} \mathrm{O}$ Ospel ${ }^{*},{ }^{2} \mathrm{~N}$ Kashani, ${ }^{3} \mathrm{~A}$ Wilson, ${ }^{4} \mathrm{~W}$ Kunz, ${ }^{5} \mathrm{P}$ Sylaia, ${ }^{6} \mathrm{~B}$ Baxter, ${ }^{7} \mathrm{~B}$ Campbell, ${ }^{8} \mathrm{U}$ Fischer, ${ }^{9} \mathrm{~A}$ Rabinstein, ${ }^{10} \mathrm{~S}$ Yoshimura, ${ }^{11} \mathrm{~J} \mathrm{HeO},{ }^{12} \mathrm{~B}$ Kim, ${ }^{13} \mathrm{M}$ Cherian, ${ }^{14} \mathrm{~F}$ Turjman, ${ }^{2} \mathrm{M}$ Foss, ${ }^{2} \mathrm{~B}$ Menon, ${ }^{15} \mathrm{G}$ Saposnik, ${ }^{2} \mathrm{M}$ Hill, ${ }^{2} \mathrm{M}$ Goyal, ${ }^{2} \mathrm{M}$ Almekhlafi. ${ }^{1}$ Radiology, University Hospital Basel, Basel, Switzerland; ${ }^{2}$ Radiology, University of Calgary, Calgary, AB, Canada; ${ }^{3}$ University of Calgary, Calgary, AB, Canada; ${ }^{4}$ Radiology, University Hospital Munich, Munich, Germany; ${ }^{5}$ Sree Chitra Tirunal Institute for Medical Sciences and Technology, Thiruvananthapuram, India; ${ }^{6}$ Radiology, Erlanger Hospital Chattanooga, Chattanooga, $T N$; ${ }^{7}$ Neurology, Royal Melbourne Hospital, University of Melbourne, Melbourne, Australia; ${ }^{8}$ Radiology, University Hospital Bern (Inselspital), Bern, Switzerland; ${ }^{9}$ Radiology, Mayo Clinic Rochester, Rochester, MN; ${ }^{10}$ Neurosurgery, Hyogo College of Medicine, Hyogo, Japan; ${ }^{11}$ Neurology, Yonsei University, Seoul, Seoul, korea, republic of; ${ }^{12}$ St. Mary's Hospital Seoul, Seoul, korea, republic of; ${ }^{13}$ Radiology, Kovai Medical center, Tamil Nadu, India; ${ }^{14}$ Radiology, Centre Hospitalier Universitaire de Lyon, Lyon, France; ${ }^{15}$ Radiology, University of Toronto, Toronto, ON, Canada

\subsection{6/neurintsurg-2019-SNIS.208}

Background Several randomized controlled trials have provided level IA evidence for the efficiency of endovascular therapy in acute ischemic stroke. We assessed the adherence to current endovascular treatment guidelines in acute ischemic stroke according to geographical region, hospital setting, medical specialty and physicians' characteristics.

Methods An international cross-sectional survey of stroke physicians and interventionalists was conducted to understand their current practice and therapy decision-making in acute stroke. Participants were randomly assigned 10 cases out of a pool of 22 scenarios and asked how they would treat the patient. Adherence to the 2018 Guidelines for the Early Management of Patients with Acute Ischemic Stroke From the American Stroke Association was analyzed, and subgroup analyses were performed for different geographical regions, hospital settings, medical subspecialty, physician experience and age. Results 607 physicians (53.6\% neurologists, 28.7\% interventionalists, $13.3 \%$ neurosurgeons, $4.7 \%$ other) from 38 countries participated in this survey. Overall guideline adherence in cases based on level of evidence $1 \mathrm{~A}$ and $2 \mathrm{~B}$ was $86.1 \%$ and $66.6 \%$. For level $1 \mathrm{~A}$ case scenarios, adherence differed 